\title{
Functional equations for Selberg zeta functions with Tate motives
}

\author{
Shin-ya Koyama* \& Nobushige Kurokawa ${ }^{\dagger}$
}

November 18, 2020

\begin{abstract}
For a compact Riemann surface $M$ of genus $g \geq 2$, we study the functional equations of the Selberg zeta functions attached with the Tate motives $f$. We prove that certain functional equations hold if and only if $f$ has the absolute automorphy.
\end{abstract}

Key Words: Selberg zeta functions, functional equations, Tate motives

AMS Subject Classifications: 11M06, 11M41, 11F72

\section{Introduction}

For a compact Riemann surface $M$ of genus $g \geq 2$ the standard Selberg zeta function $Z_{M}(s)$ is constructed as

$$
Z_{M}(s)=\prod_{P \in \operatorname{Prim}(M)} \prod_{n=0}^{\infty}\left(1-N(P)^{-s-n}\right)
$$

where $\operatorname{Prim}(M)$ denotes the set of primitive closed geodesics and the norm $N(P)$ is defined by

$$
N(P)=\exp (\text { length }(P))
$$

It has the functional equation under $s \rightarrow 1-s$ :

$$
Z_{M}(1-s)=Z_{M}(s) \exp \left((4-4 g) \int_{0}^{s-\frac{1}{2}} \pi t \tan (\pi t) d t\right) .
$$

* Department of Biomedical Engineering, Toyo University, 2100 Kujirai, Kawagoe, Saitama, 350-8585, Japan.

${ }^{\dagger}$ Department of Mathematics, Tokyo Institute of Technology, Oh-okayama, Meguro-ku, Tokyo, 152-8551, Japan. 
This functional equation was proved by Selberg [6,7] and the following symmetric version was found later:

$$
\widehat{Z}_{M}(1-s)=\widehat{Z}_{M}(s),
$$

where

$$
\widehat{Z}_{M}(s)=Z_{M}(s) \Gamma_{M}(s)
$$

with

$$
\Gamma_{M}(s)=\left(\Gamma_{2}(s) \Gamma_{2}(s+1)\right)^{2 g-2} .
$$

This double gamma function $\Gamma_{2}(s)$ is the normalized one used in $[3$ and we will recall the proof of the symmetric functional equation for $Z_{M}(s)$ in the text.

Now, the simple Euler product

$$
\zeta_{M}(s)=\prod_{P \in \operatorname{Prim}(M)}\left(1-N(P)^{-s}\right)^{-1}=\frac{Z_{M}(s+1)}{Z_{M}(s)}
$$

was also studied later and it is a more natural analog of the usual Euler product for the Riemann zeta function

$$
\zeta(s)=\prod_{p: \text { primes }}\left(1-p^{-s}\right)^{-1} .
$$

Especially the proof of the prime number theorem

$$
\pi(x) \sim \frac{x}{\log x} \quad(x \rightarrow \infty)
$$

applied to $\zeta_{M}(s)$ gives the prime geodesic theorem

$$
\pi_{M}(x) \sim \frac{x}{\log x} \quad(x \rightarrow \infty),
$$

where

$$
\pi_{M}(x)=\#\{P \in \operatorname{Prim}(M) \mid N(P) \leq x\} .
$$

The functional equation of $\zeta_{M}(s)$ has the following form:

$$
\zeta_{M}(-s)=\zeta_{M}(s)^{-1}(2 \sin (\pi s))^{4-4 g} .
$$

In this paper we study the functional equations for $\zeta_{M(f)}(s)$ with Tate motives $f$. Here we define $\zeta_{M(f)}(s)$ as

$$
\zeta_{M(f)}(s)=\prod_{k} \zeta_{M}(s-k)^{a(k)}
$$

for a Laurent polynomial

$$
f(x)=\sum_{k \in \mathbb{Z}} a(k) x^{k} \in \mathbb{Z}\left[x, x^{-1}\right] .
$$


It may be suggestive to consider $x=\mathbb{T}$ the Tate twist. Of course $\zeta_{M(1)}(s)=$ $\zeta_{M}(s)$ in our notation.

We describe results on $\zeta_{M(f)}(s)$ only for "odd" $f$ here in Introduction. See the text concerning the "even" cases.

Theorem Let $M$ and $f$ be as above. For each integer $D$ the following conditions are equivalent.

(1) $\zeta_{M(f)}(D-s)=\zeta_{M(f)}(s)$.

(2) $f\left(x^{-1}\right)=-x^{-D} f(x)$.

Remark Condition (2) is called the absolute automorphy [5]. In the paper [5] the definition of absolute automorphic forms are described in a more general setting for any function $f$ on positive real numbers, and the theory of absolute zeta functions $\zeta_{f}(s)$ is developed, which are the autmorphic $L$-functions constructed from the absolute automorphic forms $f$. It is in the framework of absolute mathematics [2,4].

For example, let $f(x)=(x-1)^{r}$ for an odd integer $r \geq 1$. Then we see that

$$
f\left(x^{-1}\right)=-x^{-r} f(x) .
$$

Hence Theorem gives the functional equation of $\zeta_{M(f)}(s)$ as

$$
\zeta_{M(f)}(r-s)=\zeta_{M(f)}(s) .
$$

A remarkable point is that we need no "gamma factors" here. In the simplest case $r=1$ we get the functional equation for

$$
\zeta_{M(f)}(s)=\frac{\zeta_{M}(s-1)}{\zeta_{M}(s)}
$$

as

$$
\zeta_{M(f)}(1-s)=\zeta_{M(f)}(s) .
$$

We remark that the study of the functional equations for

$$
Z_{M(f)}(s)=\prod_{k} Z_{M}(s-k)^{a(k)}
$$

is quite similar.

We add a few more comments on $Z_{M(f)}(s)$. Let

$$
f(x)=\sum_{k} a(k) x^{k} \in \mathbb{Z}\left[x, x^{-1}\right]
$$

satisfying

$$
f\left(x^{-1}\right)=C x^{-D} f(x)
$$


with $C= \pm 1$. Then

$$
Z_{M(f)}(s)=\prod_{k} Z_{M}(s-k)^{a(k)}
$$

has the functional equation

$$
Z_{M(f)}(D+1-s)=Z_{M(f)}(s)^{C} S_{M(f)}(s)^{C},
$$

where

$$
S_{M(f)}(s)=\prod_{k} S_{M}(s-k)^{a(k)}
$$

with

$$
S_{M}(s)=\frac{\Gamma_{M}(s)}{\Gamma_{M}(1-s)}=\left(S_{2}(s) S_{2}(s+D)\right)^{2-2 g} .
$$

Here

$$
S_{2}(s)=\frac{\Gamma_{2}(2-s)}{\Gamma_{2}(s)}
$$

is the normalized double sine function of [3]. For example $f(x)=x^{-1}-1$ $(C=-1, D=-1)$ gives the functional equation for

$$
Z_{M(f)}(s)=\frac{Z_{M}(s+1)}{Z_{M}(s)}=\zeta_{M}(s)
$$

as

$$
\zeta_{M}(-s)=\zeta_{M}(s)^{-1}(2 \sin (\pi s))^{4-4 g}
$$

where the result

$$
S_{M(f)}(s)=\frac{S_{M}(s+1)}{S_{M}(s)}=\left(\frac{S_{2}(s+2)}{S_{2}(s)}\right)^{2-2 g}=(2 \sin (\pi s))^{4-4 g}
$$

is used. Similarly we obtain the functional equation for

$$
Z_{M\left(f^{2}\right)}(s)=\frac{Z_{M}(s+2) Z_{M}(s)}{Z_{M}(s+1)}=\frac{\zeta_{M}(s+1)}{\zeta_{M}(s)}=\zeta_{M(f)}(s)
$$

as

$$
\zeta_{M(f)}(-1-s)=\zeta_{M(f)}(s)
$$

that is

$$
Z_{M\left(f^{2}\right)}(-1-s)=Z_{M\left(f^{2}\right)}(s)
$$

with no gamma factors. 


\section{Selberg zeta functions}

We describe the needed functional equations for $Z_{M}(s)$ and $\zeta_{M}(s)$ with simple proofs. Let $\Gamma_{r}(s)$ be the normalized gamma function of order $r$ defined by

$$
\Gamma_{r}(s)=\exp \left(\left.\frac{\partial}{\partial w} \zeta_{r}(w, s)\right|_{w=0}\right)
$$

with the Hurwitz zeta function of order $r$

$$
\zeta_{r}(w, s)=\sum_{n_{1}, \cdots, n_{r} \geq 0}\left(n_{1}+\cdots+n_{r}+s\right)^{-w} .
$$

The normalized sine function $S_{r}(s)$ of order $r$ is constructed as

$$
S_{r}(s)=\Gamma_{r}(s)^{-1} \Gamma_{r}(r-s)^{(-1)^{r}}:
$$

see [3] for detailed properties with proofs.

Theorem 1. (1) Let

$$
\widehat{Z}_{M}(s)=Z_{M}(s) \Gamma_{M}(s)
$$

with

$$
\Gamma_{M}(s)=\left(\Gamma_{2}(s) \Gamma_{2}(s+1)\right)^{2 g-2} .
$$

Then

$$
\widehat{Z}_{M}(1-s)=\widehat{Z}_{M}(s)
$$

$$
\zeta_{M}(-s)=\zeta_{M}(s)^{-1}(2 \sin (\pi s))^{4-4 g} .
$$

Proof. (1) From the functional equation for $Z_{M}(s)$ due to Selberg [6, 7 ]

$$
Z_{M}(1-s)=Z_{M}(s) \exp \left((4-4 g) \int_{0}^{s-\frac{1}{2}} \pi t \tan (\pi t) d t\right)
$$

we see that it is sufficient to show the identity

$$
\exp \left((4-4 g) \int_{0}^{s-\frac{1}{2}} \pi t \tan (\pi t) d t\right)=\frac{\Gamma_{M}(s)}{\Gamma_{M}(1-s)} .
$$

We first show that

$$
\exp \left((4-4 g) \int_{0}^{s-\frac{1}{2}} \pi t \tan (\pi t) d t\right)=\left(S_{2}(s) S_{2}(s+1)\right)^{2-2 g}
$$


Since both sides are equal to 1 at $s=\frac{1}{2}$ (note that $S_{2}\left(\frac{3}{2}\right)=\Gamma_{2}\left(\frac{3}{2}\right) \Gamma_{2}\left(\frac{1}{2}\right)^{-1}=$ $\left.S_{2}\left(\frac{1}{2}\right)^{-1}\right)$, it suffices to show the coincidence of logarithmic derivatives. The left hand side becomes

$$
(4-4 g) \pi\left(s-\frac{1}{2}\right) \tan \left(\pi\left(s-\frac{1}{2}\right)\right)=(2-2 g) \pi(1-2 s) \cot (\pi s) .
$$

Concerning the right hand side, the differential equation

$$
S_{2}^{\prime}(s)=\pi(1-s) \cot (\pi s) S_{2}(s)
$$

proved in 3] gives

$$
\begin{aligned}
& (2-2 g)\left(\frac{S_{2}^{\prime}(s)}{S_{2}(s)}+\frac{S_{2}^{\prime}(s+1)}{S_{2}(s+1)}\right) \\
& =(2-2 g)(\pi(1-s) \cot (\pi s)+\pi(-s) \cot (\pi(s+1))) \\
& =(2-2 g) \pi(1-2 s) \cot (\pi s) .
\end{aligned}
$$

Thus we obtain (1.1).

Next from (1.1) we get

$$
\begin{aligned}
\exp \left((4-4 g) \int_{0}^{s-\frac{1}{2}} \pi t \tan (\pi t) d t\right) & =\left(S_{2}(s) S_{2}(s+1)\right)^{2-2 g} \\
& =\left(\frac{\Gamma_{2}(2-s)}{\Gamma_{2}(s)} \cdot \frac{\Gamma_{2}(1-s)}{\Gamma_{2}(s+1)}\right)^{2-2 g} \\
& =\frac{\left(\Gamma_{2}(s) \Gamma_{2}(s+1)\right)^{2 g-2}}{\left(\Gamma_{2}(1-s) \Gamma_{2}(2-s)\right)^{2 g-2}} \\
& =\frac{\Gamma_{M}(s)}{\Gamma_{M}(1-s)} .
\end{aligned}
$$

Hence we have the functional equation

$$
Z_{M}(1-s)=Z_{M}(s) \frac{\Gamma_{M}(s)}{\Gamma_{M}(1-s)}
$$

that is

$$
\widehat{Z}_{M}(1-s)=\widehat{Z}_{M}(s)
$$

as desired.

(2) Since

$$
\zeta_{M}(s)=\frac{Z_{M}(s+1)}{Z_{M}(s)}
$$

we have

$$
\begin{aligned}
\zeta_{M}(-s) \zeta_{M}(s) & =\frac{Z_{M}(1-s)}{Z_{M}(-s)} \cdot \frac{Z_{M}(s+1)}{Z_{M}(s)} \\
& =\frac{Z_{M}(1-s)}{Z_{M}(s)} \cdot \frac{Z_{M}(s+1)}{Z_{M}(-s)} .
\end{aligned}
$$


Hence (1) gives

$$
\begin{aligned}
\zeta_{M}(-s) \zeta_{M}(s) & =\frac{\Gamma_{M}(s)}{\Gamma_{M}(1-s)} \cdot \frac{\Gamma_{M}(-s)}{\Gamma_{M}(s+1)} \\
& =\left(S_{2}(s) S_{2}(s+1)\right)^{2-2 g}\left(S_{2}(s+1) S_{2}(s+2)\right)^{2 g-2} \\
& =\left(\frac{S_{2}(s+2)}{S_{2}(s)}\right)^{2 g-2} .
\end{aligned}
$$

Recall the relations proved in [3]:

$$
\begin{aligned}
S_{2}(s+2) & =S_{2}(s+1) S_{1}(s+1)^{-1} \\
& =S_{2}(s+1)(-2 \sin (\pi s))^{-1}
\end{aligned}
$$

and

$$
\begin{aligned}
S_{2}(s) & =S_{2}(s+1) S_{1}(s) \\
& =S_{2}(s+1)(2 \sin (\pi s)) .
\end{aligned}
$$

Thus we get the functional equation for $\zeta_{M}(s)$ :

$$
\zeta_{M}(-s) \zeta_{M}(s)=(2 \sin (\pi s))^{4-4 g}
$$

that is

$$
\zeta_{M}(-s)=\zeta_{M}(s)^{-1}(2 \sin (\pi s))^{4-4 g} .
$$

\section{Functional equation for $\zeta_{M(f)}(s)$}

Let

$$
\zeta_{M(f)}(s)=\prod_{k} \zeta_{M}(s-k)^{a(k)}
$$

for

$$
f(x)=\sum_{k} a(k) x^{k} \in \mathbb{Z}\left[x, x^{-1}\right] .
$$

We prove the following theorem.

Theorem 2. For each integer $D$ the following conditions are equivalent:

(1) $\zeta_{M(f)}(D-s)=\zeta_{M(f)}(s)$.

(2) $f\left(x^{-1}\right)=-x^{-D} f(x)$.

(3) $a(D-k)=-a(k)$ for all $k$. 
Proof. We first show the equivalence $(2) \Longleftrightarrow(3)$. Let

$$
f(x)=\sum_{k} a(k) x^{k} .
$$

Then

$$
\begin{aligned}
x^{D} f\left(x^{-1}\right) & =\sum_{k} a(k) x^{D-k} \\
& =\sum_{k} a(D-k) x^{k},
\end{aligned}
$$

where we needed the exchange $k \longleftrightarrow D-k$. Hence

$$
x^{D} f\left(x^{-1}\right)=-f(x)
$$

is equivalent to

$$
a(D-k)=-a(k) \quad \text { for all } k .
$$

Next we show the equivalence $(1) \Longleftrightarrow(2)$. Since

$$
\begin{aligned}
\zeta_{M(f)}(D-s) & =\prod_{k} \zeta_{M}((D-s)-k)^{a(k)} \\
& =\prod_{k} \zeta_{M}((D-k)-s)^{a(k)} \\
& =\prod_{k} \zeta_{M}(k-s)^{a(D-k)},
\end{aligned}
$$

the functional equation for $\zeta_{M}(s)$ gives

$$
\begin{aligned}
\zeta_{M(f)}(D-s) & =\prod_{k}\left(\zeta_{M}(s-k)^{-1}(2 \sin (\pi s))^{4-4 g}\right)^{a(D-k)} \\
& =\left(\prod_{k} \zeta_{M}(s-k)^{-a(D-k)}\right)(2 \sin (\pi s))^{(4-4 g) f(1)},
\end{aligned}
$$

where we used

$$
f(1)=\sum_{k} a(k)=\sum_{k} a(D-k) .
$$

Hence we have the following expression

$$
\frac{\zeta_{M(f)}(D-s)}{\zeta_{M(f)}(s)}=\left(\prod_{k} \zeta_{M}(s-k)^{-a(D-k)-a(k)}\right)(2 \sin (\pi s))^{(4-4 g) f(1)} .
$$

From this expression the equivalence $(1) \Longleftrightarrow(3)$ is shown as follows. First the condition (3) (or equivalently (2)) implies $f(1)=0$ and that $a(D-k)+a(k)=0$ for all $k$. Hence (2.1) gives

$$
\frac{\zeta_{M(f)}(D-s)}{\zeta_{M(f)}(s)}=1
$$


which is (1).

Now assume (1). Then from (2.1) we have the identity

$$
\prod_{k} \zeta_{M}(s-k)^{a(D-k)+a(k)}=(2 \sin (\pi s))^{(4-4 g) f(1)} .
$$

Since $\zeta_{M}(s)$ is non-zero holomorphic in $\operatorname{Re}(s)>1$, the left hand side of (2.2) is non-zero holomorphic for sufficiently large $\operatorname{Re}(s)$. Hence looking at the left hand side at sufficiently large $s \in \mathbb{Z}$ we see $f(1)=0$. Then (2.2) gives

$$
\prod_{k} \zeta_{M}(s-k)^{a(D-k)+a(k)}=1 .
$$

We remark that (2.3) is actually written as

$$
\prod_{k \leq K} \zeta_{M}(s-k)^{a(D-k)+a(k)}=1
$$

for some $K \in \mathbb{Z}$, since $f(x) \in \mathbb{Z}\left[x, x^{-1}\right]$. Hence we have the identity

$$
\zeta_{M}(s-K)^{a(D-K)+a(K)}=\prod_{k<K} \zeta_{M}(s-k)^{-a(D-k)-a(k)} .
$$

Look at (2.5) at $s=K+1$, then the right hand side is

$$
\prod_{k<K} \zeta_{M}(1+(K-k))^{-a(D-k)-a(k)},
$$

which is a finite non-zero value. Hence looking at the left hand side of (2.5) we see that

$$
a(D-K)+a(K)=0
$$

since $\zeta_{M}(s-K)$ has a simple pole at $s=K+1$. Thus (2.5) becomes

$$
\prod_{k \leq K-1} \zeta_{M}(s-k)^{a(D-k)+a(k)}=1
$$

Inductively we see (3).

Theorem 2 treated "odd" $f$. The next theorem deals with the other case for "even" $f$.

Theorem 3. For each integer $D$ the following conditions are equivalent:

(1) $\zeta_{M(f)}(D-s)=\zeta_{M(f)}(s)^{-1}(2 \sin (\pi s))^{(4-4 g) f(1)}$.

(2) $f\left(x^{-1}\right)=x^{-D} f(x)$.

(3) $a(D-k)=a(k)$ for all $k$. 
Proof. The equivalence $(2) \Longleftrightarrow(3)$ is shown exactly as in the proof of Theorem 2. Now we show $(1) \Longleftrightarrow(3)$. Notice that

$$
\begin{aligned}
\zeta_{M(f)}(D-s) & =\prod_{k} \zeta_{M}((D-s)-k)^{a(k)} \\
& =\prod_{k} \zeta_{M}((D-k)-s)^{a(k)} \\
& =\prod_{k} \zeta_{M}(k-s)^{a(D-k)} \\
& =\prod_{k}\left(\zeta_{M}(s-k)^{-1}(2 \sin (\pi s))^{4-4 g}\right)^{a(D-k)} \\
& =\left(\prod_{k} \zeta_{M}(s-k)^{-a(D-k)}\right)(2 \sin (\pi s))^{(4-4 g) f(1)}
\end{aligned}
$$

where we used that

$$
\sum_{k} a(D-k)=f(1)
$$

Proof of (3) $\Longrightarrow(1)$. From (3) we have

$$
\begin{aligned}
\zeta_{M(f)}(D-s) & =\left(\prod_{k} \zeta_{M}(s-k)^{-a(k)}\right)(2 \sin (\pi s))^{(4-4 g) f(1)} \\
& =\zeta_{M(f)}(s)^{-1}(2 \sin (\pi s))^{(4-4 g) f(1)},
\end{aligned}
$$

which is (1).

Proof of $(1) \Longrightarrow(3)$. Since

$$
\zeta_{M(f)}(D-s)=\left(\prod_{k} \zeta_{M}(s-k)^{-a(D-k)}\right)(2 \sin (\pi s))^{(4-4 g) f(1)}
$$

as above, we have

$$
\frac{\zeta_{M(f)}(D-s)}{\zeta_{M(f)}(s)^{-1}(2 \sin (\pi s))^{(4-4 g) f(1)}}=\prod_{k} \zeta_{M}(s-k)^{a(k)-a(D-k)} .
$$

Hence from the assumption (1) we get

$$
\prod_{k} \zeta_{M}(s-k)^{a(k)-a(D-k)}=1,
$$

which can be written as

$$
\prod_{k \leq K} \zeta_{M}(s-k)^{a(k)-a(D-k)}=1
$$


that is

$$
\zeta_{M}(s-K)^{a(K)-a(D-K)}=\prod_{k<K} \zeta_{M}(s-k)^{a(D-k)-a(k)} .
$$

Then we obtain $a(D-K)=a(K)$ and inductively $a(D-k)=a(k)$ for all $k$ exactly as in the proof of Theorem 2 .

Example. Let $f(x)=(x-1)^{r}$ for an even integer $r \geq 0$. Then we see that

$$
f\left(x^{-1}\right)=x^{-r} f(x) .
$$

Hence we obtain the functional equation

$$
\zeta_{M(f)}(r-s)=\zeta_{M(f)}(s)^{-1} \times \begin{cases}(2 \sin (\pi s))^{4-4 g} & (r=0) \\ 1 & (r \geq 2, \text { even })\end{cases}
$$

Of course the $r=0$ case gives the functional equation of $\zeta_{M}(s)$.

Remark. Let $f(x)=(x-1)^{r}$ for an integer $r \geq 0$. Then $\zeta_{M}(s)$ is written explicitly as

$$
\zeta_{M(f)}(s)=\prod_{k=0}^{r} \zeta_{M}(s-k)^{(-1)^{r-k}\left(\begin{array}{l}
r \\
k
\end{array}\right)} .
$$

In this case another suggestive notation would be

$$
\zeta_{M(f)}(s)=\zeta_{M \otimes \mathbb{G}_{m}^{r}}(s)
$$

since $(x-1)^{r}$ is the counting function of $\mathbb{G}_{m}^{r}$; see [1,2,4].

\section{References}

[1] A. Connes and C. Consani, Schemes over $\mathbb{F}_{1}$ and zeta functions, Compositio Math. 146 (2010), 1383-1415.

[2] A. Deitmar, S. Koyama, and N. Kurokawa, Counting and zeta functions over $\mathbb{F}_{1}$, Abh. Math. Seminar Univ. Hamburg 85 (2015), 59-71.

[3] S. Koyama and N. Kurokawa, Multiple sine functions, Forum Math. 15 (2003), 839-876.

[4] N. Kurokawa and H. Ochiai, Dualities for absolute zeta functions and multiple gamma functions, Proc. Japan Acad. 89A (2013), 75-79.

[5] N. Kurokawa and H. Tanaka, Absolute zeta functions and the automorphy, Kodai Math. J. 40 (2017), 584-614.

[6] A. Selberg, Harmonic analysis and discontinuous groups in weakly symmetric Riemannian spaces with applications to Dirichlet series, J. Indian Math. Soc. 20 (1956), 47-87.

[7] _ Göttingen lectures, Collected Works, Vol. I, 1989, pp. 626-674. 